\title{
Dyspnea caused by a giant retroperitoneal liposarcoma: A case report
}

\author{
ARGYRIOS IOANNIDIS ${ }^{1}$, CHRISTOS KOUTSERIMPAS ${ }^{1}$, MICHAEL KONSTANTINIDIS ${ }^{2}$, \\ IOANNIS DRIKOS $^{1}$, PANAGIOTIS VOULGARIS ${ }^{1}$ and NIKOLAOS ECONOMOU ${ }^{1}$ \\ ${ }^{1}$ Department of General Surgery, 'Sismanoglion' General Hospital of Attika, 15126 Athens; \\ ${ }^{2}$ National and Kapodistrian University of Athens, School of Medicine, 11527 Athens, Greece
}

Received April 11,2018; Accepted May 8, 2018

DOI: $10.3892 / \mathrm{ol} .2018 .8791$

\begin{abstract}
Liposarcomas are the most common soft tissue tumors with various histological subtypes. They usually appear in the retroperitoneal region of the abdomen, but their symptomatology remains unclear and their diagnosis, as well as treatment challenging. A case of a 55-year-old female patient with dyspnea and light diffuse abdominal pain caused by a giant retroperitoneal liposarcoma is presented. The patient had an unremarkable medical history, while the computed tomography scan revealed a large mass at the right portion of the abdomen, with its upper limits to the lower edge and the gate portion of the liver. The mass was in contact with the right kidney, the inferior vena cava and the right renal vein, causing mild dilation of the right kidney pelvis. Without any evidence of intra-abdominal metastases, the tumor was surgically resected. The histological analysis of the tumor revealed a well-differentiated liposarcoma. The patient had an uneventful recovery and was discharged on the 10th postoperative day. Until today (4 years later) she remains asymptomatic, without any signs of recurrence. The retroperitoneal liposarcoma is a clinical entity with unclear clinical symptoms and the physician should consider including it in the differential diagnosis of a majority of symptoms, such as dyspnea.
\end{abstract}

\section{Introduction}

Liposarcomas are the most common soft tissue tumors, presenting with a variety of symptoms such as bleeding, weight loss and abdominal pain. They are usually located in the retroperitoneal region (1). The incidence of retroperitoneal liposarcomas vary between 0.07 and $0.2 \%$ of all retroperitoneal tumors, while $85 \%$ of these tumors are considered

Correspondence to: Dr Argyrios Ioannidis, Department of General Surgery, 'Sismanoglion' General Hospital of Attika, 1 Sismanoglou Street, 15126 Athens, Greece

E-mail: agis.ioannidis@gmail.com

Key words: giant liposarcoma, retroperitoneal tumors, abdominal pain, dyspnea malignant (2). A rare case of a 55-year-old female patient with a giant retroperitoneal liposarcoma presented with dyspnea is reported in the present study.

\section{Case report}

A 55-year-old female, suffering from dyspnea and light diffuse abdominal pain was referred to our hospital. The symptoms had begun 2 weeks prior to admission. Her past medical history was unremarkable. The patient was stable (blood pressure, 135/950 mmHg; heart rate, 85 beats/min; saturation, $94 \%$; and breathing rate, 13 breaths $/ \mathrm{min})$ and afebrile $\left(36.7^{\circ} \mathrm{C}\right)$. Clinical examination revealed distended abdomen and a palpable mass extending from the epigastrium to the right pelvic region. The laboratory results showed hematocrit $(\mathrm{Hct})=31.2 \%$, and hemoglobin $(\mathrm{Hgb})=10,9 \mathrm{~g} / \mathrm{dl}$.

Plain X-ray views of the abdomen showed deviation of the bowels to the left side. Further imaging with computed tomography $(\mathrm{CT})$ scan, as well as magnetic imaging resonance (MRI) exhibited a solid mass located in the right abdomen, with its central region not absorbing the intravenous contrast, which was attributed to central necrosis or cystic degeneration (Fig. 1). The mass occupied the right portion of the abdomen repulsing sections of the gastrointestinal tract, with its upper borders to the lower edge and gate portion of the liver and lower borders to the right ovary. The size of this mass was calculated to be $23 \times 19 \times 12 \mathrm{~cm}$. The position of the mass was suppressing the anatomical structures and organs away from their natural position. In addition, the tumor was in contact with the right kidney, the inferior vena cava and the right renal vein causing mild dilation of the right pelvis of the kidney. In the pelvis, the mass was in contact with the bladder, the sigmoid colon, as well as the right iliac vessels which were verified as non-obstructed by the compatible intravenous contrast analysis. There was no evidence of intra-abdominal or peritoneal metastases.

Surgical excision of the mass was performed through a midline incision (Fig. 2). The patient did not suffer any postoperative complications, had an uneventful recovery and was discharged on the 6th postoperative day. Pathological examination revealed a well-differentiated liposarcoma, without evidence of cellular atypia, while loose fibrous connective tissue between the malignant cells was observed. 

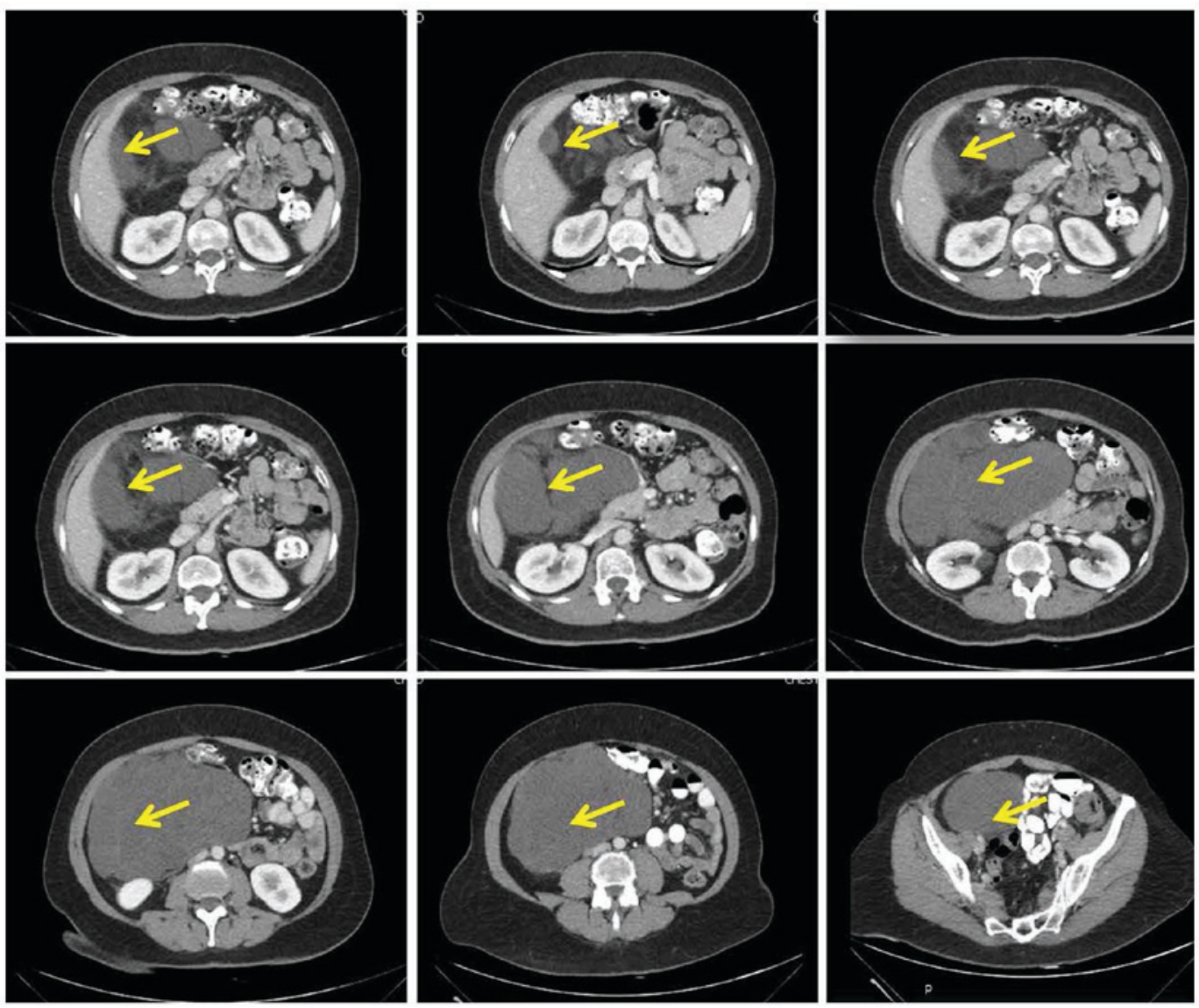

Figure 1. Retroperitoneal liposarcoma appearing as a solid mass (arrow), with its central region not absorbing dye attributed, due to central necrosis or cystic degeneration. The mass was found as a cystic tumor of the retroperitoneal area which extends from the lower edge of the liver to the right ovary and pelvis region.

The patient was followed up in the out-patient clinic with physical examination and imaging (chest/abdominal/pelvic CT) every 6 months for 3 years and then annually for a total of 4 years until today.

\section{Discussion}

Retroperitoneal sarcomas are rare malignancies accounting for approximately $15 \%$ of all sarcoma cases, while their incidence is $0.3-0.4 \%$ per 100,000 individuals $(1,2)$. Liposarcomas account for approximately $40 \%$ of retroperitoneal sarcomas making them the most common type, followed by leiomyosarcoma and malignant fibrous histiocytoma, while it is estimated that they comprise $0.07-0.2 \%$ of all neoplasms (3-5).

Retroperitoneal liposarcomas have a higher incidence in the sixth and seventh decade, while the influence of sex remains unclear $(3,5)$. The present case was a 55-year-old female. About $50 \%$ of cases are diagnosed when their size is over $20 \mathrm{~cm}$. The reported case sought medical assistance 2 weeks after the onset of symptoms, while the mass was already $23 \times 19 \times 12 \mathrm{~cm}$. This is due to the fact that the retroperitoneal region offers large spaces for expansion and the symptomatology may present in the late stages $(3,5)$. Additionally, symptoms are usually non-specific, such as flank or abdominal pain, lower extremity swelling or pain, constipation or urinary symptoms $(3,5)$. The reported case suffered mainly from dyspnea, a symptom very rarely associated with these tumors. Of note, most retroperitoneal liposarcomas remain asymptomatic.

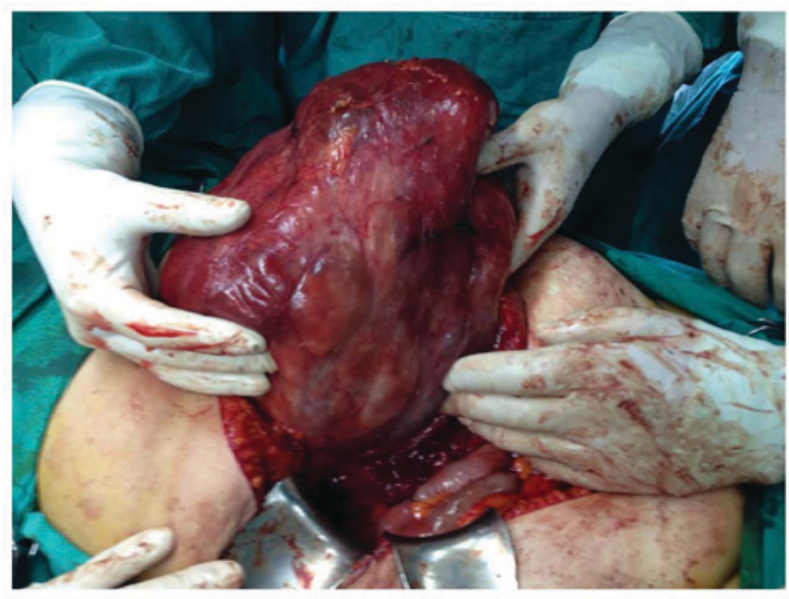

Figure 2. Intraoperative image of the resected specimen.

Liposarcomas have 5 types: Well-differentiated liposarcoma, dedifferentiated liposarcoma, myxoid/round cell liposarcoma, pleomorphic liposarcoma and mixed liposarcoma. Well-differentiated and dedifferentiated liposarcomas are the most commonly observed retroperitoneal ones, while well-differentiated liposarcoma has 3 subtypes: Lipomatous, sclerotic and inflammatory liposarcoma $(3,6-8)$. The reported case is a well-differentiated liposarcoma, which is usually a slow-growing mass with minimum metastatic behavior. Dedifferentiated liposarcomas are either primary or develop 
from a well-differentiated one, while myxoid/round cell liposarcomas have a high metastatic potential and are aggressive. Mixed liposarcoma are the rarest and have a poor prognosis $(3,7)$.

In patients suspected with retroperitoneal sarcoma a pre-treatment biopsy is not usually performed. Retroperitoneal masses are more likely to be liposarcomas than lipomas and therefore it is best to surgically excise them. In patients unfit for surgery or those who are candidates for preoperative radiotherapy and/or chemotherapy or those with metastases a fine-needle aspiration or core-needle biopsy should be performed. In cases that the management would change with a definite diagnosis and percutaneous biopsies have failed, open biopsy should be considered $(3,4)$.

Regarding management of these tumors, surgical excision represents the definite treatment, while surgical resection with negative microscopic margins is the most consistent prognostic factor (3,9-16). Surgical treatment of these cases is challenging, since many structures and organs are usually involved and adipocyte differentiation makes it difficult to distinguish the tumor from retroperitoneal fat tissue (17). Additionally, some cases involve unresectable vital structures or resection could lead to increased morbidity (18). In these cases, a biopsy should be considered and preoperative radiotherapy and/or chemotherapy should be performed to preoperatively downstage the tumor (3). Studies evaluating the aggressive surgical resection have reported good results with 5-year local recurrence-free and disease-specific survival rates of 55 and $75 \%$, respectively (12). Due to the potential high difficulty of these operations the open approach is usually preferred. In the reported patient an open approach with midline incision was performed and the tumor was resected with negative microscopic margins (19).

Recurrence of these tumors usually occurs within 6 months to 2 years following surgical excision with local recurrence being much more common than distant metastases. Five-year local recurrence rates following complete macroscopic resection are approximately $50 \%$ for well-differentiated retroperitoneal liposarcomas. Early diagnosed and operated recurrences have complete excision rates in approximately $90 \%$ of cases (20). Therefore, it is crucial to regularly follow up these patients with imaging, such as CT scan and/or MRI (21). It is recommended that patients with low-grade retroperitoneal soft-tissue sarcomas, successfully treated with surgical resection, should have a follow-up physical examination with imaging (chest/abdominal/pelvic $\mathrm{CT}$ ) every 3 to 6 months for 2 to 3 years, then annually, while for those with high-grade sarcoma, successfully treated with surgical resection, a follow-up physical examination with imaging (chest/abdominal/pelvic CT) every 3 to 6 months for 2 to 3 years, then every 6 months for the next 2 years, and then annually is recommended. The present patient has been followed up (4 years now), according to this protocol, without any signs of recurrence up-to-date (22).

In conclusion, retroperitoneal sarcomas are relatively rare tumors that represent a surgical challenge. Surgical resection with negative microscopic margins is the definite treatment. It is of note that the symptomatology is non-specific. The reported patient presented with dyspnea, something extremely rare and not usually reported in the literature. It is therefore crucial for the physician to perform a thorough physical examination and to keep a keen eye for cases such as this.

\section{Acknowledgements}

Not applicable.

\section{Funding}

No funding was received.

\section{Availability of data and materials}

The datasets used and/or analyzed during the current study are available from the corresponding author on reasonable request.

\section{Authors' contributions}

AI and CK were involved in the conception of the study. AI, MK and SA performed the review. AI, NM and KK analyzed and interpreted the data. AI, MK and KK wrote the manuscript. NM and KK critically revised the manuscript. All authors read and approved the final manuscript.

\section{Ethics approval and consent to participate}

Not applicable.

\section{Consent for publication}

The patient provided written informed consent for the publication of any associated data and accompanying images.

\section{Competing interests}

The authors declare that they have no competing interests.

\section{References}

1. Mettlin C, Priore R, Rao U, Gamble D, Lane W and Murphy P: Results of the national soft-tissue sarcoma registry. J Surg Oncol 19: 224-227, 1982.

2. Herrera-Gómez A, Ortega-Gutiérrez C, Betancourt AM and Luna-Ortiz K: Giant retroperitoneal liposarcoma. World J Surg Oncol 6: 115, 2008.

3. Vijay A and Ram L: Retroperitoneal liposarcoma: A comprehensive review. Am J Clin Oncol 38: 213-219, 2015.

4. Das Gupta TK: Tumors and tumor-like conditions of the adipose tissue. Curr Probl Surg 7: 1-60, 1970.

5. Chouairy CJ, Abdul-Karim FW and MacLennan GT: Retroperitoneal liposarcoma. J Urol 177: 1145, 2007.

6. Hosaka A, Masaki Y, Yamasaki K, Aoki F, Sugizaki K and Ito E: Retroperitoneal mixed-type liposarcoma showing features of four different subtypes. Am Surg 74: 1202-1205, 2008.

7. Sheffield BS and Nielsen TO: Myxoid liposarcoma in a 91-year-old patient. Mol Cytogenet 6: 50, 2013.

8. Fritz B, Schubert F, Wrobel G, Schwaenen C, Wessendorf S, Nessling M, Korz C, Rieker RJ, Montgomery K, Kucherlapati R, et al: Microarray-based copy number and expression profiling in dedifferentiated and pleomorphic liposarcoma. Cancer Res 62: 2993-2998, 2002.

9. Lewis JJ, Leung D, Woodruff JM and Brennan MF: Retroperitoneal soft-tissue sarcoma: Analysis of 500 patients treated and followed at a single institution. Ann Surg 228: 355-365, 1998. 
10. Lee SY, Goh BK, Teo MC, Chew MH, Chow PK, Wong WK, Ooi LL and Soo KC: Retroperitoneal liposarcomas: The experience of a tertiary Asian center. World J Surg Oncol 9: 12-17, 2011

11. Bonvalot S, Rivoire M, Castaing M, Stoeckle E, Le Cesne A, Blay JY and Laplanche A: Primary retroperitoneal sarcomas: A multivariate analysis of surgical factors associated with local control. J Clin Oncol 27: 31-37, 2009.

12. Strauss DC, Hayes AJ, Thway K, Moskovic EC, Fisher C and Thomas JM: Surgical management of primary retroperitoneal sarcoma. Br J Surg 97: 698-706, 2010.

13. van Dalen T, Plooij JM, van Coevorden F, van Geel AN, Hoekstra HJ, Albus-Lutter Ch, Slootweg PJ and Hennipman A; Dutch Soft Tissue Sarcoma Group: Long-term prognosis of primary retroperitoneal soft tissue sarcoma. Eur J Surg Oncol 33: 234-238, 2007.

14. Alldinger I, Yang Q, Pilarsky C, Saeger HD, Knoefel WT and Peiper M: Retroperitoneal soft tissue sarcomas: Prognosis and treatment of primary and recurrent disease in 117 patients. Anticancer Res 26 (2B): 1577-1581, 2006.

15. Erzen D, Sencar M and Novak J: Retroperitoneal sarcoma: 25 years of experience with aggressive surgical treatment at the Institute of Oncology, Ljubljana. J Surg Oncol 91: 1-9, 2005.

16. Singer S, Corson JM, Demetri GD, Healey EA, Marcus K and Eberlein TJ: Prognostic factors predictive of survival for truncal and retroperitoneal soft-tissue sarcoma. Ann Surg 221: 185-195, 1995.

17. Elias A, Ryan L, Sulkes A, Collins J, Aisner J and Antman KH: Response to mesna, doxorubicin, ifosfamide, and dacarbazine in 108 patients with metastatic or unresectable sarcoma and no prior chemotherapy. J Clin Oncol 7: 1208-1216, 1989.
18. Shibata D, Lewis JJ, Leung DH and Brennan MF: Is there a role for incomplete resection in the management of retroperitoneal liposarcomas? J Am Coll Surg 193: 373-379, 2001.

19. Shalhav AL, Chan SW, Bercowsky E, Elbahnassy AM, McDougall EM and Clayman RV: Laparoscopic exploration in the management of retroperitoneal masses. JSLS 3: 209-214, 1999.

20. Kim EY, Kim SJ, Choi D, Lee SJ, Kim SH, Lim HK and Song SY: Recurrence of retroperitoneal liposarcoma: Imaging findings and growth rates at follow-up CT. AJR Am J Roentgenol 191: 1841-1846, 2008.

21. Song T, Shen J, Liang BL, Mai WW, Li Y and Guo HC: Retroperitoneal liposarcoma: MR characteristics and pathological correlative analysis. Abdom Imaging 32: 668-674, 2007.

22. NCCN: Clinical Practice Guidelines in Oncology. http://www. nccn.org/professionals/physician_gls/PDF/sarcoma.pdf. Accessed April 8, 2018 